KEK Preprint 97-22

DPNU-97-26

May 1997

\title{
Quark Mass Matrices in Superstring Models
}

\author{
Tatsuo Kobayashi 7 \\ Institute of Particle and Nuclear Studies, \\ High Energy Accelerator Research Organization, Tanashi, Tokyo 188, Japan \\ Zhi-zhong Xing [ \\ Department of Physics, Nagoya University, Chikusa-ku, Nagoya 464-01, Japan
}

\begin{abstract}
Four simple but realistic patterns of quark mass matrices are derived from orbifold models of superstring theory in the absence of gauge symmetries. Two of them correspond to the Ramond-Roberts-Ross types, which have five texture zeros in up and down quark sectors. The other two, with four texture zeros, preserve the structural parallelism between up and down sectors. The phenomenological consequences of these mass matrices on flavor mixings and $C P$ violation are analyzed at the weak scale. With the same input values of quark mass ratios, we find that only one or two of the four patterns can be in good agreement with current experimental data.
\end{abstract}

(Accepted for publication in Int. J. Mod. Phys. A)

* Electronic address: Kobayast@tanashi.kek.jp

$\dagger$ Electronic address: Xing@eken.phys.nagoya-u.ac.jp 


\section{Introduction}

The origin of fermion masses is one of the most important problems in particle physics. To date much work has been devoted to understanding this puzzle. One of the plausible mechanisms for fermion mass generation is to use nonrenormalizable couplings such as $H Q_{i} q_{j}(\theta / M)^{n_{i j}}$. When suitable fields like $\theta$ develop their vacuum expectation values (VEVs), these nonrenormalizable couplings could provide effective Yukawa couplings $H Q_{i} q_{j}(\langle\theta\rangle / M)^{n_{i j}}$ with a hierarchical structure [1]. A realistic hierarchy can be derived from certain types of structures of the power parameters $n_{i j}$.

The structure of these powers is determined by underlying theories with underlying symmetries. For example, gauge symmetries as well as global symmetries can be used to derive fermion mass matrices [1, 2, 3].

Superstring theory is a promising candidate for the unified theory of all interactions including gravity. Superstring theory has proper selection rules for nonrenormalizable couplings in addition to gauge symmetries of field theory. These proper symmetries in superstring theory, stringy symmetries, are originated from structure of a six-dimensional compactified space of string vacua. They can lead to realistic fermion mass matrices, which may differ from those derived from gauge symmetries f. Actually some efforts have been made to derive fermion mass matrices by use of stringy symmetries in Refs. [6]- [10]. Refs. [6, 7] discuss fermionic construction of 4D string models and Calabi-Yau models, where stringy symmetries are discrete symmetries. In comparison, Refs. [9, 10] discuss orbifold models, which have complicated selection rules [11, 12]. A systematic study of possible patterns of quark mass matrices that can be derived from stringy symmetries, however, has been lacking in the literature.

In this work we investigate various possibilities to obtain simple but realistic quark mass matrices from orbifold models by nontrivially extending the analysis in Refs. [9, 10], where only one type of symmetric quark mass matrices was discussed. Following the principle of simplicity, here we concentrate only on symmetric quark mass matrices with five or four texture zeros. It has been shown by Ramond, Roberts and Ross (RRR) that symmetric quark mass matrices with more than five texture zeros are not realistic [13], i.e., they cannot fit current experimental data on quark mixings and $C P$ violation. Note that symmetric quark mass matrices with five texture zeros can be classified into five types, the so-called RRR patterns [13]. We show that only two of the five RRR patterns can be naturally derived from orbifold models. Since mass matrices with four texture zeros include more degrees of freedom,

\footnotetext{
${ }^{\ddagger}$ In the approach of gauge symmetries sfermion masses gain D-term contributions to soft scalar masses through supersymmetry breaking $[$. Such D-term contributions should be treated carefully so that they do not break degeneracy between squark masses, as required by present experiments of flavor changing neutral currents $[5$.
} 
we further impose the up-down structural parallelism on them to obtain relatively simple patterns. Then we can find that there exist only two types of realistic quark mass matrices with four texture zeros and up-down parallel structures. The phenomenological consequences of the obtained quark mass matrices are also analyzed, from the string scale to the weak scale, by use of renormalization-group equations in the framework of the minimal supersymmetric standard model (MSSM).

The remaining parts of this paper are organized as follows. In section 2 we first give a brief review of selection rules of orbifold models. Using the selection rules of couplings we derive some typical patterns of quark mass matrices, and then calculate their mass eigenvalues and flavor mixing matrices at the string scale. In section 3 the obtained patterns of mass matrices are confronted with current experimental data at the weak scale. Section 4 is devoted to a summary with some concluding remarks.

\section{Quark mass matrices in orbifold models}

\section{A. Orbifold models}

The orbifold construction is one of the simplest and most interesting constructions to derive four-dimensional string vacua [14]. In orbifold models, string states consist of the bosonic string on the four-dimensional space-time and a six-dimensional orbifold, as well as their right-moving superpartners and left-moving gauge parts. The right-moving fermionic parts are bosonized, and momenta of bosonized fields span an $\mathrm{SO}(10)$ lattice. An orbifold is obtained through the division of a six-dimensional space $R^{6}$ by a six-dimensional lattice and its automorphism $\theta$. Closed strings on the orbifold are classified into untwisted and twisted types. For the $\theta^{k}$-twisted sector $T_{k}$, the string coordinate on the orbifold, $x_{\nu}(\nu=1 \sim 6)$, satisfies the following boundary condition:

$$
x_{\nu}(\sigma=2 \pi)=\left(\theta^{k} x\right)_{\nu}(\sigma=0)+e_{\nu}
$$

where $e_{\nu}$ is a lattice vector. A zero-mode of this string satisfies the same condition as Eq. (2.1) and it is called a fixed point. The fixed point is represented in terms of its space group element $\left(\theta^{k}, e_{\nu}\right)$; and we have the corresponding six-dimensional ground state $\left|\left(\theta^{k}, e_{\nu}\right)\right\rangle$. All fixed points in $T_{k}$ are not fixed under $\theta$. To obtain $\theta$-eigenstates, we have to take linear combinations of states corresponding to fixed points of $\theta^{k}$ as 115, 16]

$$
\left|\left(\theta^{k}, e_{\nu}\right)\right\rangle+\gamma^{-1}\left|\theta\left(\theta^{k}, e_{\nu}\right)\right\rangle+\cdots+\gamma^{-(m-1)}\left|\theta^{m-1}\left(\theta^{k}, e_{\nu}\right)\right\rangle
$$

Here $\theta^{m}$ denotes the smallest twist fixing $\left(\theta^{k}, e_{\nu}\right)$ itself. Thus we have $m<k$. These linear combinations have eigenvalues $\gamma=\exp [\mathrm{i} 2 \pi n / m]$ under the $\theta$-twist with an integer $n$. We 
denote the $T_{k}$ sector with the $\theta$-eigenvalue $\gamma$ as $T_{k(\gamma)}$, when we specify the $\theta$-eigenvalue. We take a complex basis $\left(X_{i}, \bar{X}_{i}\right)(i=1 \sim 3)$ for the compactified space, e.g., $X_{1}=x_{1}+\mathrm{i} x_{2}$. Oscillated states in $T_{k}$ are created by $\partial X_{i(k)}$ and $\partial \bar{X}_{i(k)}$ on the ground states given in (2.2). The twisted sectors have shifted $\mathrm{SO}(10)$ momenta. Every shifted $\mathrm{SO}(10)$ momentum of massless $T_{k}$ states has been shown explicitly in Refs. [15, 16].

Couplings are calculated by using vertex operators $V_{a}$ corresponding to states [17, 18]. Vertex operators consist of several parts, i.e., the four-dimensional part, the six-dimensional ground state of the $T_{k}$ sector as (2.2), oscillators on it, the bosonized $\mathrm{SO}(10)$ part, the gauge part, and the ghost part. Nonvanishing couplings are invariant under a symmetry of each part. Coupling terms are allowed if they are gauge invariant and space-group invariant. The latter implies that a product of space-group elements $\left(\theta_{a}^{k}, e_{a}\right)$ for coupling states should satisfy $\prod_{a}\left(\theta_{a}^{k}, e_{a}\right)=(1,0)$ up to the conjugacy class [. Furthermore, a product of eigenvalues $\gamma_{a}$ should satisfy $\prod_{a} \gamma_{a}=1$. In addition, the $\mathrm{SO}(10)$ momentum and the ghost number should be conserved. The corresponding correlation function $\left\langle V_{1} \cdots V_{n}\right\rangle$ should be invariant under a $Z_{N}$ rotation of oscillators as

$$
\partial X_{i(k)} \rightarrow e^{\mathrm{i} 2 \pi k v^{i}} \partial X_{i(k)}
$$

where $e^{\mathrm{i} 2 \pi v^{i}}$ are eigenvalues of $\theta$ in the complex basis $X_{i}$. Note that even vertex operators corresponding to non-oscillated massless states include oscillators, when we change their pictures from the -1 or $-1 / 2$ picture [17]. These selection rules have been discussed in Ref. [12].

$Z_{6}$-II orbifold models have most twisted sectors among $Z_{N}$ orbifold models, whose sixdimensional orbifolds are constructed as products of two-dimensional orbifolds. Thus one can expect that $Z_{6}$-II orbifold models are simpler but possess various types of couplings, which could be useful to obtain realistic mass matrices. For this reason, we concentrate our attention upon $Z_{6}$-II orbifold models in the following.

The $Z_{6}$-II orbifold has eigenvalues $v_{i}=(2,1,-3) / 6$. Massless matter states in $Z_{6}$-II orbifold models correspond to $T_{1}, T_{2}, T_{3}$ and $T_{4}$ sectors [15, 16, 19]. The other twisted sectors correspond only to antimatter states. Allowed nonrenormalizable couplings in $Z_{6}$-II orbifold models are listed in Table 1, where PGI means the point group invariance.

\section{B. Mass matrices with up-down parallelism}

In general, the underlying theory like supergravity or superstring theory has nonrenormalizable couplings as

$$
h_{\mathrm{u} i j} H_{2} Q_{i} u_{j}\left(\theta_{\mathrm{u}} / M_{2}\right)^{n_{i j}}, \quad h_{\mathrm{d} i j} H_{1} Q_{i} d_{j}\left(\theta_{\mathrm{d}} / M_{1}\right)^{n_{i j}^{\prime}},
$$

${ }^{\dagger}$ For the selection rule due to the space group, see Ref. [16] for some details. 
Table 1: Allowed nonrenormalizable couplings in $Z_{6}$-II orbifold models.

\begin{tabular}{lc}
\hline \hline Coupling & Condition \\
\hline$T_{2}^{3 \ell} T_{3}^{2 m}$ & $\ell>0, m>0$ \\
$T_{4}^{3 \ell} T_{3}^{2 m}$ & $\ell>0, m>0$ \\
$T_{1}^{2 \ell} T_{2}^{m} T_{4}^{n}$ & $\ell=2 p+1,2 \ell+2 m+n=3 q, \ell>0, m>0, n>0$, PGI \\
$T_{1}^{\ell} T_{2}^{m} T_{3}^{n} T_{4}^{p}$ & $\ell>0, n>0, m$ or $p>0$, PGI \\
\hline \hline
\end{tabular}

where $Q_{i}$ is the $\mathrm{SU}(2)$ doublet of quark fields, $u_{j}\left(d_{j}\right)$ denotes the up-type (down-type) $\mathrm{SU}(2)$ singlet of quark fields, and $H_{2,1}$ are the Higgs fields for the up and down sectors. Here $h_{\mathrm{u} i j}$ and $h_{\mathrm{d} i j}$ denote coupling strengths, which can be calculated within the framework of superstring theory. Their magnitudes are of $O(1)$ in most cases. When the fields $\theta_{\mathrm{u}, \mathrm{d}}$ develop VEVs, these couplings become Yukawa couplings with suppression factors $\varepsilon_{\mathrm{u}}=\left(\left\langle\theta_{\mathrm{u}}\right\rangle / M_{2}\right)^{n_{i j}}$ and $\varepsilon_{\mathrm{d}}=\left(\left\langle\theta_{\mathrm{d}}\right\rangle / M_{1}\right)^{n_{i j}^{\prime}}$. They can lead to a hierarchical structure in the fermion mass matrices. In general, we expect $\varepsilon_{\mathrm{u}} \neq \varepsilon_{\mathrm{d}}$. For example, the mixing between light and heavy Higgs fields leads to $\varepsilon_{\mathrm{u}} \neq \varepsilon_{\mathrm{d}}$ [2, [13].

Using allowed nonrenormalizable couplings in $Z_{6}$-II orbifold models, we can obtain simple and realistic quark mass matrices. We first study the possibility for symmetric quark mass matrices with up-down structural parallelism and four texture zeros. Here the texture zero does not mean that the matrix element is completely vanishing; instead it means that the matrix element is remarkably suppressed or vanishingly small.

We assign Higgs fields $H_{1}$ and $H_{2}$ to the $T_{4(\gamma=1)}$ sector. The first, second and third families of both up and down quarks are assigned to $T_{2(\gamma=1)}, T_{3(\gamma=1)}$ and $T_{1}$, respectively. In this assignment we assume fields with $T_{1}, T_{2(\gamma=-1)}$ and $T_{4(\gamma=-1)}$ sectors to develop VEVs. Then we obtain the following quark mass matrices [9, [10]:

$$
\text { Pattern S1 : } \quad M_{\mathrm{u}, \mathrm{d}}=c_{\mathrm{u}, \mathrm{d}}\left(\begin{array}{ccc}
0 & \varepsilon_{\mathrm{u}, \mathrm{d}}^{3} & 0 \\
\varepsilon_{\mathrm{u}, \mathrm{d}}^{3} & \varepsilon_{\mathrm{u}, \mathrm{d}}^{2} & \varepsilon_{\mathrm{u}, \mathrm{d}}^{2} \\
0 & \varepsilon_{\mathrm{u}, \mathrm{d}}^{2} & 1
\end{array}\right)
$$


with $h_{\mathrm{u} i j} / h_{\mathrm{u} 33}\left(h_{\mathrm{d} i j} / h_{\mathrm{d} 33}\right)$ of $O(1)$. Here $c_{\mathrm{u}}=h_{\mathrm{u} 33}\left\langle H_{2}\right\rangle$ and $c_{\mathrm{d}}=h_{\mathrm{d} 33}\left\langle H_{1}\right\rangle$. The $(1,2),(2,2)$ and $(2,3)$ elements are originated from $\left(T_{2} T_{3} T_{4}\right) T_{1}^{3},\left(T_{3}^{2} T_{4}\right) T_{4(\gamma=-1)}^{2}$ and $\left(T_{1} T_{3} T_{4}\right) T_{2(\gamma=-1)}^{2}$ couplings, respectively. The $(1,1)$ or $(1,3)$ element does not completely vanish, but it is sufficiently suppressed in comparison with its neighboring elements. For example, the $(1,3)$ element can be obtained as $\varepsilon_{\mathrm{u}, \mathrm{d}}^{9}$ by $\left(T_{1} T_{2} T_{4}\right) T_{1}^{9}$, if we assign fixed points in a certain way.

We are able to obtain another type of quark mass matrices with the up-down parallel structure. We assign both of $H_{1}$ and $H_{2}$ to $T_{4(\gamma=1)}$, and assign the first, second and third families of up and down quarks to $T_{3(\gamma=1)}, T_{3(\gamma=\omega)}$ and $T_{1}$, respectively, where $\omega=e^{\mathrm{i} 2 \pi / 3}$. In this assignment, $T_{1}, T_{3(\gamma=\omega)}$ and $T_{4(\gamma=1)}$ fields are assumed to develop VEVs. Then we get quark mass matrices of the type

$$
\text { Pattern S2: } \quad M_{\mathrm{u}, \mathrm{d}}=c_{\mathrm{u}, \mathrm{d}}\left(\begin{array}{ccc}
0 & \varepsilon_{\mathrm{u}, \mathrm{d}}^{4} & 0 \\
\varepsilon_{\mathrm{u}, \mathrm{d}}^{4} & \varepsilon_{\mathrm{u}, \mathrm{d}}^{3} & \varepsilon_{\mathrm{u}, \mathrm{d}}^{3} \\
0 & \varepsilon_{\mathrm{u}, \mathrm{d}}^{3} & 1
\end{array}\right) .
$$

Here the $(2,2)$ and $(2,3)$ elements are originated from $T_{1} T_{3(\gamma=\omega)}^{3} T_{4(\gamma=1)}^{2}$ coupling, while the $(1,2)$ element is due to $T_{3(\gamma=1)} T_{3(\gamma=\omega)}^{3} T_{4(\gamma=1)}^{3}$ coupling.

Note that those quark mass matrices with four texture zeros in entries different from $(1,1)$ and $(1,3)$ are phenomenologically unrealistic. Thus patterns S1 and S2 should be the only candidates of realistic and simple quark mass matrices with four texture zeros and up-down structural parallelism. Giving up the up-down structural parallelism, one can indeed derive many patterns of quark mass matrices with four texture zeros. Such a work is less interesting in both theory and phenomenology, because there may exist much simpler patterns with five texture zeros [13] (as one can see later on).

\section{Mass matrices of Ramond-Roberts-Ross types}

Five types of symmetric quark mass matrices with five texture zeros, the so-called RRR patterns, have been obtained in Ref. [13]. The down mass matrices in three RRR patterns take the form like (2.6). Thus one can in principle derive these three RRR patterns from orbiford models by choosing the appropriate assignment for the up-type quarks.

For example, we can assign $u, c$ and $t$ quarks to $T_{3(\gamma=\omega)}, T_{3\left(\gamma=\omega^{2}\right)}$ and $T_{1}$, respectively, without changing the assignment of other fields. Then we have

$$
\text { Pattern A1: } \quad M_{\mathrm{u}}=c_{\mathrm{u}}\left(\begin{array}{ccc}
0 & \varepsilon_{\mathrm{u}}^{3} & 0 \\
\varepsilon_{\mathrm{u}}^{3} & \varepsilon_{\mathrm{u}}^{2} & 0 \\
0 & 0 & 1
\end{array}\right), \quad M_{\mathrm{d}}=c_{\mathrm{d}}\left(\begin{array}{ccc}
0 & \varepsilon_{\mathrm{d}}^{4} & 0 \\
\varepsilon_{\mathrm{d}}^{4} & \varepsilon_{\mathrm{d}}^{3} & \varepsilon_{\mathrm{d}}^{3} \\
0 & \varepsilon_{\mathrm{d}}^{3} & 1
\end{array}\right) \text {. }
$$

This pattern corresponds to the first RRR-type mass matrices [13]. The $(2,2)$ element in $M_{\mathrm{u}}$ is originated from $T_{3(\gamma=\omega)} T_{3\left(\gamma=\omega^{2}\right)} T_{4} T_{1}^{2}$ and/or $T_{3(\gamma=\omega)} T_{3\left(\gamma=\omega^{2}\right)} T_{4}^{3}$; while the $(1,2)$ and $(2,1)$ 
elements are due to $T_{3(\gamma=\omega)}^{3} T_{4}^{2} T_{1}$ and $T_{3} T_{3(\gamma=\omega)} T_{3\left(\gamma=\omega^{2}\right)} T_{4}^{2} T_{1}$, respectively. The other elements of $M_{\mathrm{u}}$, e.g., the $(2,3)$ and $(3,2)$ elements, can be sufficiently suppressed if fixed points are chosen in a proper way.

One can also obtain the third RRR-type mass matrices in a similar way. For example, $u$, $c$ and $t$ quarks may be assigned to $T_{3(\gamma=1)}, T_{3\left(\gamma=\omega^{2}\right)}$ and $T_{1}$, respectively, without changing the assignment of other fields. The resultant quark mass matrices take the form

$$
\text { Pattern A2: } \quad M_{\mathrm{u}}=c_{\mathrm{u}}\left(\begin{array}{ccc}
0 & 0 & \varepsilon_{\mathrm{u}}^{3} \\
0 & \varepsilon_{\mathrm{u}}^{3} & 0 \\
\varepsilon_{\mathrm{u}}^{3} & 0 & 1
\end{array}\right), \quad M_{\mathrm{d}}=c_{\mathrm{d}}\left(\begin{array}{ccc}
0 & \varepsilon_{\mathrm{d}}^{4} & 0 \\
\varepsilon_{\mathrm{d}}^{4} & \varepsilon_{\mathrm{d}}^{3} & \varepsilon_{\mathrm{d}}^{3} \\
0 & \varepsilon_{\mathrm{d}}^{3} & 1
\end{array}\right) \text {. }
$$

Here the $(2,2)$ and $(1,3)$ elements are originated from $T_{3\left(\gamma=\omega^{2}\right)} T_{3(\gamma=\omega)} T_{4} T_{1}^{2}$ and $T_{1}^{2} T_{3(\gamma=1)}^{2} T_{4}$ couplings, respectively. The other matrix elements in $M_{\mathrm{u}}$ may be remarkably suppressed through a proper choice of fixed points.

It is difficult to obtain the other three RRR patterns of mass matrices from $Z_{6}-$ II orbifold models. One obvious reason is that those three patterns involve more complicated hierarchies. For example, the up mass matrix in the fourth RRR pattern reads [13]:

$$
M_{\mathrm{u}} \sim c_{\mathrm{u}}\left(\begin{array}{ccc}
0 & \lambda^{6} & 0 \\
\lambda^{6} & \lambda^{4} & \lambda^{2} \\
0 & \lambda^{2} & 1
\end{array}\right)
$$

where $\lambda=0.22$ is the Cabibbo angle. Clearly $M_{\mathrm{u}}$ in (2.9) contains five hierarchies: $1, \lambda^{2}, \lambda^{4}$, $\lambda^{6}$ and 0 . Such a complicated hierarchical structure cannot be easily derived from orbifold models by only using stringy selection rules, because the number of twisted sectors is limited. In the above-mentioned mechanism of $Z_{6}$-II orbifold models, it is also difficult to make the $(2,2)$ element of a mass matrix vanishingly small in comparison with its nonvanishing $(2,3)$ and $(3,2)$ elements. For example, there is not a straightforward way to reproduce the second RRR pattern which includes the following up mass matrix [13]:

$$
M_{\mathrm{u}} \sim c_{\mathrm{u}}\left(\begin{array}{ccc}
0 & \lambda^{6} & 0 \\
\lambda^{6} & 0 & \lambda^{2} \\
0 & \lambda^{2} & 1
\end{array}\right)
$$

Similarly, we find that the popular Fritzsch-type mass matrices [20] cannot be easily derived from string models by purely using stringy selection rules.

\section{Mass eigenvalues and flavor mixings}

In this subsection we first calculate mass eigenvalues for the four types of quark mass matrices obtained above, and then derive the corresponding flavor mixing matrices at the string scale $M_{S}$. 
Up to now we have taken $h_{\mathrm{u} i j} / h_{\mathrm{u} 33}=1$ and $h_{\mathrm{d} i j} / h_{\mathrm{d} 33}=1$. Within the framework of superstring theory we can calculate magnitudes of these couplings. The coupling strengths $h_{\mathrm{u} i j}\left(\right.$ or $\left.h_{\mathrm{d} i j}\right)$ are obtained as $h_{\mathrm{u} i j} \sim \exp \left(-a_{i j} T\right)$ (or $h_{\mathrm{d} i j} \sim \exp \left(-a_{i j}^{\prime} T\right)$ ), where $T$ is the moduli parameter representing the size of six-dimensional compactified space and $a_{i j}$ (or $a_{i j}^{\prime}$ ) is a constant depending on the combination of fixed points for couplings [18 3. The factors $\exp \left(-a_{i j} T\right)$ in the mass matrix elements are generally different from one another. These factors seem to be of $O(1)$. Note that the $(1,2)$ and $(2,3)$ elements of Pattern S1 have different powers of $\varepsilon_{\mathrm{u}, \mathrm{d}}$ even in the absence of $h_{\mathrm{u} i j}\left(\right.$ or $\left.h_{\mathrm{d} i j}\right)$, thus the effect of $h_{\mathrm{u} 12} / h_{\mathrm{u} 23}\left(\right.$ or $\left.h_{\mathrm{d} 12} / h_{\mathrm{d} 23}\right)$ is not important. Indeed the deviation of $h_{\mathrm{u} 12} / h_{\mathrm{u} 23}$ (or $h_{\mathrm{d} 12} / h_{\mathrm{d} 23}$ ) from unity can be absorbed by a redefinition of the basic parameter $\varepsilon_{\mathrm{u}}\left(\right.$ or $\left.\varepsilon_{\mathrm{d}}\right)$. In contrast, the $(2,2)$ and $(2,3)$ elements in Pattern S1 have the same power of $\varepsilon_{\mathrm{u}, \mathrm{d}}$, implying that the effect of $h_{\mathrm{u} 22} / h_{\mathrm{u} 23}\left(\right.$ or $\left.h_{\mathrm{d} 22} / h_{\mathrm{d} 23}\right)$ might be non-negligible. Hence we introduce two factors $\omega_{\mathrm{u}, \mathrm{d}}$ of $O(1)$ for the $(2,2)$ elements of $M_{\mathrm{u}, \mathrm{d}}$ in Pattern $\mathrm{S} 1$, so as to signify the underlying difference between $h_{\mathrm{u} 22}$ (or $h_{\mathrm{d} 22}$ ) and $h_{\mathrm{u} 23}$ (or $h_{\mathrm{d} 23}$ ). It is reasonable to take $h_{\mathrm{u} i j} / h_{\mathrm{u} 33}=1$ and $h_{\mathrm{d} i j} / h_{\mathrm{d} 33}=1$ for other elements of Pattern S1. Phenomenologically we find that such a free parameter $\left(\omega_{\mathrm{u}}\right.$ or $\left.\omega_{\mathrm{d}}\right)$ may be crucial for us to properly reproduce the quark mass eigenvalues from $M_{\mathrm{u}}$ or $M_{\mathrm{d}}$ [10]. For the same reason, we introduce factors $\omega_{\mathrm{u}, \mathrm{d}}$ for the $(2,2)$ elements of all the above-derived quark mass matrices except $M_{\mathrm{u}}$ of Pattern A1, where the $(1,2)$ and $(2,2)$ elements have got different powers of $\varepsilon_{\mathrm{u}}$. Furthermore, we assume all parameters in $M_{\mathrm{u}, \mathrm{d}}$ (i.e., $c_{\mathrm{u}, \mathrm{d}}, \varepsilon_{\mathrm{u}, \mathrm{d}}$ and $\omega_{\mathrm{u}, \mathrm{d}}$ ) to be real. Our analytical results for quark mass eigenvalues, in leading order approximations, are listed in Table 2.

To calculate the mixing matrix of quark flavors, we need introduce a simple phase matrix

$$
P=\left(\begin{array}{ccc}
1 & 0 & 0 \\
0 & e^{\mathrm{i} \phi} & 0 \\
0 & 0 & e^{\mathrm{i} \phi}
\end{array}\right),
$$

where $\phi$ denotes the possible phase difference between $M_{\mathrm{u}}$ and $M_{\mathrm{d}}$. Such a $C P$-violating phase may arise from the dynamical details of our fermion mass generation mechanism, e.g., the background antisymmetric tensors in orbifold models or imaginary VEVs of $\theta$. Phenomenologically the existence of $\phi$ is necessary for the Ansatz to properly reproduce the Cabibbo angle and $C P$ violation. The flavor mixing matrix, i.e., the Cabibbo-Kobayashi-Maskawa (CKM) matrix, is given by

$$
V \equiv O_{\mathrm{u}}^{\mathrm{T}} P O_{\mathrm{d}}
$$

where $O_{\mathrm{u}}$ and $O_{\mathrm{d}}$ are the orthogonal matrices diagonalizing $M_{\mathrm{u}}$ and $M_{\mathrm{d}}$, respectively. Due to the hierarchy of quark masses, it is easy to show that all the above four patterns lead to

\footnotetext{
${ }^{\S}$ Similarly the $C P$-violating phases can be introduced into some elements in the case of nonvanishing background antisymmetric tensors [21], although without such antisymmetric tensors $C P$ is a nice symmetry of superstring - the relevant couplings are always real except trivial phases [22].
} 
Table 2: Quark mass eigenvalues derived from four types of quark mass matrices.

\begin{tabular}{lcccccc}
\hline \hline & $m_{t}$ & $m_{c}$ & $m_{u}$ & $m_{b}$ & $m_{s}$ & $m_{d}$ \\
\hline Pattern & $c_{\mathrm{u}}$ & $\varepsilon_{\mathrm{u}}^{2} \omega_{\mathrm{u}} c_{\mathrm{u}}$ & $\frac{\varepsilon_{\mathrm{u}}^{4}}{\omega_{\mathrm{u}}} c_{\mathrm{u}}$ & $c_{\mathrm{d}}$ & $\varepsilon_{\mathrm{d}}^{2} \omega_{\mathrm{d}} c_{\mathrm{d}}$ & $\frac{\varepsilon_{\mathrm{d}}^{4}}{\omega_{\mathrm{d}}} c_{\mathrm{d}}$ \\
$\mathrm{S} 1$ & $c_{\mathrm{u}}$ & $\varepsilon_{\mathrm{u}}^{3} \omega_{\mathrm{u}} c_{\mathrm{u}}$ & $\frac{\varepsilon_{\mathrm{u}}^{5}}{\omega_{\mathrm{u}}} c_{\mathrm{u}}$ & $c_{\mathrm{d}}$ & $\varepsilon_{\mathrm{d}}^{3} \omega_{\mathrm{d}} c_{\mathrm{d}}$ & $\frac{\varepsilon_{\mathrm{d}}^{5}}{\omega_{\mathrm{d}}} c_{\mathrm{d}}$ \\
$\mathrm{S} 2$ & & & & & & \\
$\mathrm{~A} 1$ & $c_{\mathrm{u}}$ & $\varepsilon_{\mathrm{u}}^{2} c_{\mathrm{u}}$ & $\varepsilon_{\mathrm{u}}^{4} c_{\mathrm{u}}$ & $c_{\mathrm{d}}$ & $\varepsilon_{\mathrm{d}}^{3} \omega_{\mathrm{d}} c_{\mathrm{d}}$ & $\frac{\varepsilon_{\mathrm{d}}^{5}}{\omega_{\mathrm{d}}} c_{\mathrm{d}}$ \\
& & & & & & \\
$\mathrm{A} 2$ & $c_{\mathrm{u}}$ & $\varepsilon_{\mathrm{u}}^{3} \omega_{\mathrm{u}} c_{\mathrm{u}}$ & $\varepsilon_{\mathrm{u}}^{6} c_{\mathrm{u}}$ & $c_{\mathrm{d}}$ & $\varepsilon_{\mathrm{d}}^{3} \omega_{\mathrm{d}} c_{\mathrm{d}}$ & $\frac{\varepsilon_{\mathrm{d}}^{5}}{\omega_{\mathrm{d}}} c_{\mathrm{d}}$ \\
\hline \hline
\end{tabular}

$\left|V_{u d}\right| \approx\left|V_{c s}\right| \approx\left|V_{t b}\right| \approx 1$ in leading order approximations. In addition, we can reproduce the results $\left|V_{u s}\right| \approx\left|V_{c d}\right|$ and $\left|V_{c b}\right| \approx\left|V_{t s}\right|$, which hold at both low and high energy scales [23] (as indicated by current data and unitarity of $V$ [24, 25]). The analytical results for $\left|V_{u s}\right|,\left|V_{c b}\right|$, $\left|V_{u b} / V_{c b}\right|$ and $\left|V_{t d} / V_{t s}\right|$ are listed in Table 3 .

\section{Quark mixings at the weak scale}

Now let us confront the above-obtained flavor mixing matrix $V$ with low-energy experimental data, in order to phenomenologically "justify" the string-inspired quark mass matrices $M_{\mathrm{u}, \mathrm{d}}$. For this purpose, we have to run the elements of $V$ from the string scale $M_{S}\left(\sim 10^{17} \mathrm{GeV}\right)$ to the weak scale $M_{Z}\left(\sim 10^{2} \mathrm{GeV}\right)$. Theoretically it is instructive to assume the minimal supersymmetric standard model (MSSM) below $M_{S}$ 10. Then one can apply the renormalization-group equations to $M_{\mathrm{u}, \mathrm{d}}$ and $V$ in the framework of the MSSM.

\section{A. Scale-independent results}

The one-loop renormalization group equations for quark mass ratios and flavor mixing matrix elements have been explicitly presented in Ref. [26]. In view of the hierarchy of Yukawa couplings and quark mixing angles, one can make reliable analytical approximations 
Table 3: Leading order results for CKM matrix elements $\left|V_{u s}\right|,\left|V_{c b}\right|,\left|V_{u b}\right|$ and $\left|V_{t d}\right|$.

\begin{tabular}{|c|c|c|c|c|}
\hline Pattern & $\left|V_{u s}\right|$ & $\left|V_{c b}\right|$ & $\left|\frac{V_{u b}}{V_{c b}}\right|$ & $\left|\frac{V_{t d}}{V_{t s}}\right|$ \\
\hline S1 & $\left|\frac{\varepsilon_{\mathrm{u}}}{\omega_{\mathrm{u}}} e^{\mathrm{i} \phi}-\frac{\varepsilon_{\mathrm{d}}}{\omega_{\mathrm{d}}}\right|$ & $\left|\varepsilon_{\mathrm{u}}^{2}-\varepsilon_{\mathrm{d}}^{2}\right|$ & $\left|\frac{\varepsilon_{\mathrm{u}}}{\omega_{\mathrm{u}}}\right|$ & $\left|\frac{\varepsilon_{\mathrm{d}}}{\omega_{\mathrm{d}}}\right|$ \\
\hline S2 & $\left|\frac{\varepsilon_{\mathrm{u}}}{\omega_{\mathrm{u}}} e^{\mathrm{i} \phi}-\frac{\varepsilon_{\mathrm{d}}}{\omega_{\mathrm{d}}}\right|$ & $\left|\varepsilon_{\mathrm{u}}^{3}-\varepsilon_{\mathrm{d}}^{3}\right|$ & $\left|\frac{\varepsilon_{\mathrm{u}}}{\omega_{\mathrm{u}}}\right|$ & $\left|\frac{\varepsilon_{\mathrm{d}}}{\omega_{\mathrm{d}}}\right|$ \\
\hline A1 & $\left|\varepsilon_{\mathrm{u}} e^{\mathrm{i} \phi}-\frac{\varepsilon_{\mathrm{d}}}{\omega_{\mathrm{d}}}\right|$ & $\left|\varepsilon_{\mathrm{d}}^{3}\right|$ & $\left|\varepsilon_{\mathrm{u}}\right|$ & $\left|\frac{\varepsilon_{\mathrm{d}}}{\omega_{\mathrm{d}}}\right|$ \\
\hline A2 & $\left|\frac{\varepsilon_{\mathrm{d}}}{\omega_{\mathrm{d}}}\right|$ & $\left|\varepsilon_{\mathrm{d}}^{3}\right|$ & $\left|\frac{\varepsilon_{\mathrm{u}}^{3}}{\omega_{\mathrm{d}} \varepsilon_{\mathrm{d}}^{3}}\right|$ & $\left|\frac{\varepsilon_{\mathrm{d}}}{\omega_{\mathrm{d}}} e^{\mathrm{i} \phi}+\frac{\varepsilon_{\mathrm{u}}^{3}}{\varepsilon_{\mathrm{d}}^{3}}\right|$ \\
\hline
\end{tabular}

for the relevant evolution equations by keeping only the leading terms. It is straightforward to find that the running effects of $m_{u} / m_{c}$ and $m_{d} / m_{s}$ are negligibly small. The evolutions of $\left|V_{u s}\right|$ and $\left|V_{c d}\right|$ involve the second-family Yukawa couplings, thus they can be safely neglected. In addition, the diagonal elements of $V$ have negligible evolutions with energy. Only $\left|V_{u b}\right|,\left|V_{c b}\right|$, $\left|V_{t d}\right|$ and $\left|V_{t s}\right|$ may have significant renormalization-group effects, and their running behaviors are indeed identical in leading order approximations [26].

Taking the above points into account, we find that $\varepsilon_{\mathrm{u}} / \omega_{\mathrm{u}} \approx \sqrt{m_{u} / m_{c}}$ and $\varepsilon_{\mathrm{d}} / \omega_{\mathrm{d}} \approx \sqrt{m_{d} / m_{s}}$ in Pattern S1 are approximately scale-independent. So are the parameters $\varepsilon_{\mathrm{u}} / \omega_{\mathrm{u}} \approx \sqrt{m_{u} / m_{c}}$ and $\varepsilon_{\mathrm{d}} / \omega_{\mathrm{d}} \approx \sqrt{m_{d} / m_{s}}$ in Pattern S2; $\varepsilon_{\mathrm{u}} \approx \sqrt{m_{u} / m_{c}}$ and $\varepsilon_{\mathrm{d}} / \omega_{\mathrm{d}} \approx \sqrt{m_{d} / m_{s}}$ in Pattern A1; $\varepsilon_{\mathrm{u}}^{3} / \omega_{\mathrm{u}} \approx m_{u} / m_{c}$ and $\varepsilon_{\mathrm{d}} / \omega_{\mathrm{d}} \approx \sqrt{m_{d} / m_{s}}$ in Pattern A2. Consequently some scale-independent results for quark mixings at the weak scale $M_{Z}$ can be straightforwardly obtained, as listed in Table 4.

Some discussions about Table 4 are in order:

(a) For Pattern $\mathrm{S} 1, \mathrm{~S} 2$ or A1, the $C P$-violating phase $\phi$ can be determined from the present data on $\left|V_{u s}\right|$ :

$$
\phi \approx \arccos \left[\frac{1}{2} \sqrt{\frac{m_{c} m_{s}}{m_{u} m_{d}}}\left(\frac{m_{u}}{m_{c}}+\frac{m_{d}}{m_{s}}-\left|V_{u s}\right|^{2}\right)\right] .
$$


Table 4: Leading order results for $\left|V_{u s}\right|,\left|V_{u b} / V_{c b}\right|$ and $\left|V_{t d} / V_{t s}\right|$ at $M_{Z}$.

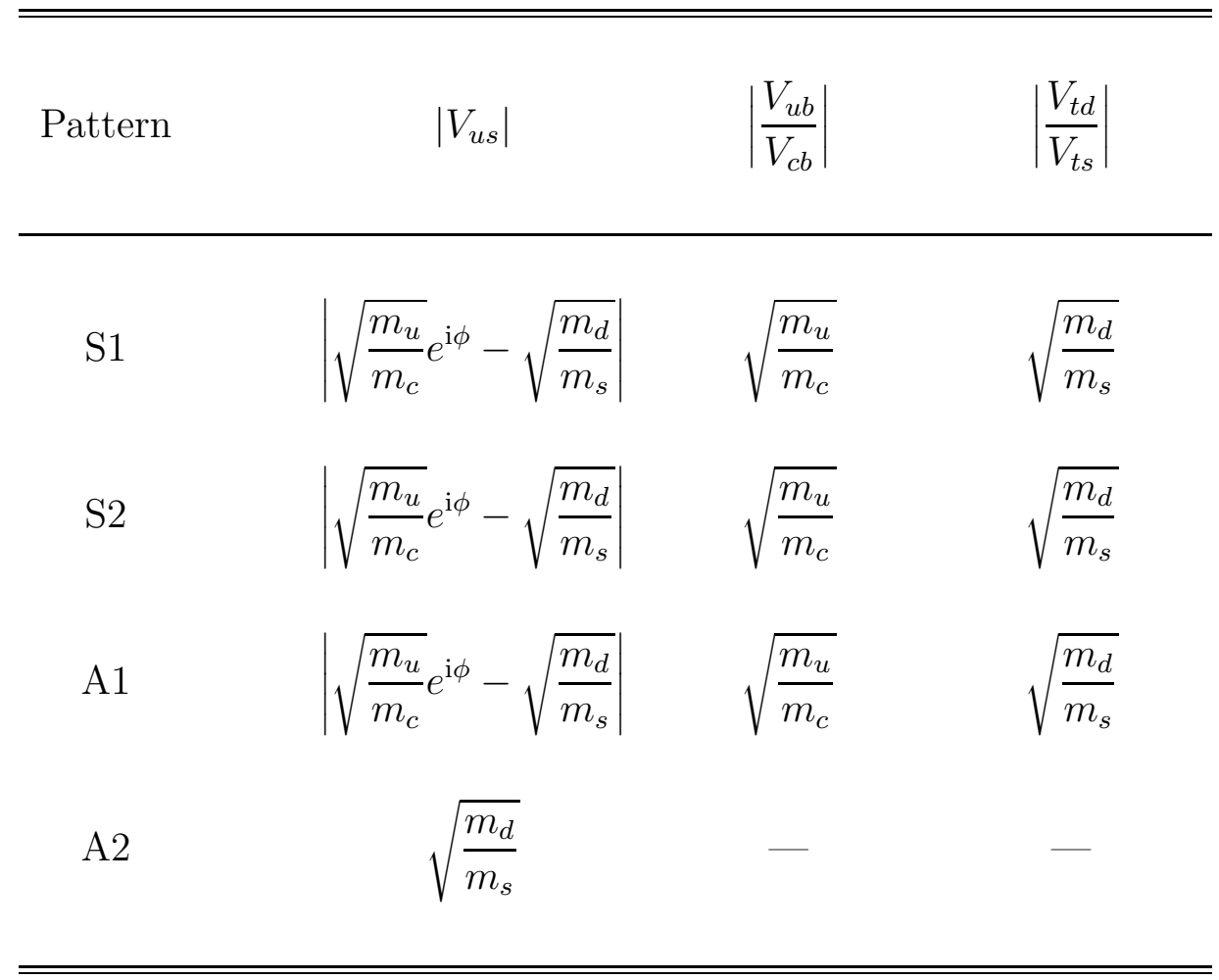

By use of $\left|V_{u s}\right| \exp =0.2205 \pm 0.0018$ [24], $m_{s} / m_{d}=19.3 \pm 0.9$ [27] and $10^{-3} \leq m_{u} / m_{c} \leq 10^{-2}$ [24], we calculate $\phi$ and find an allowed region for its value in the first quadrant: $75^{0} \leq \phi \leq 85^{0}$. Current data on $C P$ violation in $K^{0}-\bar{K}^{0}$ mixing (i.e., $\epsilon_{K}$ ) 24 have excluded the possibility of $\phi<0$.

(b) For Pattern A2, we get $\left|V_{u s}\right| \approx \sqrt{m_{d} / m_{s}} \approx 0.228 \pm 0.005$, in agreement with the present experimental value $\left|V_{u s}\right|_{\exp }=0.2205 \pm 0.0018$. In this pattern, the $C P$-violating phase is in principle determinable from $\left|V_{t d} / V_{t s}\right|$.

(c) The instructive relations $\left|V_{u b} / V_{c b}\right| \approx \sqrt{m_{u} / m_{c}}$ and $\left|V_{t d} / V_{t s}\right| \approx \sqrt{m_{d} / m_{s}}$ hold approximately in Patterns S1, S2 and A1. They result from the texture zeros of $(1,1),(1,3)$ and $(3,1)$ elements for both up and down mass matrices [20, 28, 29]. Typically taking $m_{u}=5.1 \pm 0.9$ $\mathrm{MeV}$ and $m_{c}=1.3 \mathrm{GeV}$ at the scale $\mu=1 \mathrm{GeV}$ [30, we predict $\left|V_{u b} / V_{c b}\right|=0.063 \pm 0.005$ and $\left|V_{t d} / V_{t s}\right|=0.228 \pm 0.005$. In comparison, current measurements have given $\left|V_{u b} / V_{c b}\right|_{\exp }=$ $0.08 \pm 0.02$ [24] and constrained the ratio of $\left|V_{t d}\right|$ to $\left|V_{t s}\right|$ to be in the region $0.15-0.34$ [31].

\section{B. Scale-dependent results}

For all four patterns of quark mass matrices, the analytical expressions of $\left|V_{c b}\right|$ and $\left|V_{t s}\right|$ obtained in Table 3 will be altered due to non-negligible running effects from the string scale 
$\mu=M_{S}$ to the weak scale $\mu=M_{Z}$. The results for $\left|V_{u b} / V_{c b}\right|$ and $\left|V_{t d} / V_{t s}\right|$ in Pattern A2 are also sensitive to the renormalization-group effects, since they depend strongly upon the mass ratios $m_{c} / m_{t}$ and $m_{s} / m_{b}$. The relevant evolution functions can be defined by [32]

$$
\xi_{t, b}=\exp \left[-\frac{1}{16 \pi^{2}} \int_{0}^{\ln \left(M_{S} / M_{Z}\right)} f_{t, b}^{2}(\chi) \mathrm{d} \chi\right]
$$

where $\chi \equiv \ln \left(\mu / M_{Z}\right)$; and $f_{t}$ and $f_{b}$ are Yukawa coupling eigenvalues of the top and bottom quarks, respectively. A good approximation is that the third-family Yukawa couplings of quarks (and charged leptons), together with the gauge couplings, play the dominant roles in the renormalization-group equations [26]. Then the magnitudes of $\xi_{t}$ and $\xi_{b}$ can be evaluated for arbitrary $\tan \beta$ (the ratio of Higgs vacuum expectation values in the MSSM) from $M_{S}$ to $M_{Z}$, and the numerical results have been given in [32]. In terms of $\xi_{t}$ and $\xi_{b}$, three key evolution relations based on the MSSM read [32]:

$$
\begin{aligned}
\left.\frac{m_{s}}{m_{b}}\right|_{M_{Z}} & =\left.\frac{1}{\xi_{t} \xi_{b}^{3}} \frac{m_{s}}{m_{b}}\right|_{M_{S}}, \\
\left.\frac{m_{c}}{m_{t}}\right|_{M_{Z}} & =\left.\frac{1}{\xi_{t}^{3} \xi_{b}} \frac{m_{c}}{m_{t}}\right|_{M_{S}}, \\
\left|V_{c b}\right|_{M_{Z}} & =\frac{1}{\xi_{t} \xi_{b}}\left|V_{c b}\right|_{M_{S}} .
\end{aligned}
$$

The running behaviors of $\left|V_{u b}\right|,\left|V_{t d}\right|$ and $\left|V_{t s}\right|$ are identical to that of $\left|V_{c b}\right|$. By use of Eq. (3.3), we are able to obtain the renormalized expressions of $\left|V_{c b}\right|$ at $M_{Z}$, as listed in Table 5 .

At the weak scale $M_{Z}$, the ratios $\left|V_{u b} / V_{c b}\right|$ and $\left|V_{t d} / V_{t s}\right|$ obtained in Pattern A2 can be renormalized as

$$
\left|\frac{V_{u b}}{V_{c b}}\right| \approx\left(\xi_{t}^{1 / 2} \xi_{b}^{-5 / 2}\right)\left(\frac{m_{u}}{m_{c}}\right)^{1 / 2}\left(\frac{m_{c}}{m_{t}}\right)^{1 / 2}\left(\frac{m_{s}}{m_{b}}\right)^{-1}
$$

and

$$
\left|\frac{V_{t d}}{V_{t s}}\right| \approx\left|\left(\frac{m_{d}}{m_{s}}\right)^{1 / 2} e^{\mathrm{i} \phi}+\left(\xi_{t}^{3 / 4} \xi_{b}^{-7 / 4}\right)\left(\frac{m_{u}}{m_{c}}\right)^{1 / 2}\left(\frac{m_{d}}{m_{s}}\right)^{-3 / 8}\left(\frac{m_{c}}{m_{t}}\right)^{1 / 2}\left(\frac{m_{s}}{m_{b}}\right)^{-3 / 4}\right|
$$

respectively. We observe that these two analytical expressions, although their quantitative results could be compatible with current data, are qualitatively less interesting than those simpler ones derived from Patterns S1, S2 and A1.

For the purpose of illustration, we make an estimation of the above scale-dependent $\left|V_{c b}\right|$, $\left|V_{u b} / V_{c b}\right|$ and $\left|V_{t d} / V_{t s}\right|$. We take $m_{u} / m_{c}=0.004, m_{c} / m_{t}=0.005, m_{d} / m_{s}=0.05$ and $m_{s} / m_{b}=$ 0.035 at $M_{Z}$ typically [24, 30]. With the help of the numerical results of $\xi_{t}$ and $\xi_{b}$ obtained in Ref. [32], we plot the renormalized $\left|V_{c b}\right|$ as a function of $\tan \beta$ in Fig. 1 for all four patterns of quark mass matrices; and illustrate the renormalized $\left|V_{u b} / V_{c b}\right|$ and $\left|V_{t d} / V_{t s}\right|$ changing with $\tan \beta$ in Fig. 2 for Pattern A2. Some comments on Figs. 1 and 2 are in order.

(1) The uncertainty of $\left|V_{c b}\right|$, arising from the unknown value of $\tan \beta$, may be as large as 0.01. This error is obviously larger than the experimental error in determining $\left|V_{c b}\right|$ (e.g., 
Table 5: Leading order results for $\left|V_{c b}\right|$ at the weak scale $M_{Z}$.

\begin{tabular}{cc}
\hline \hline Pattern & Renormalized $\left|V_{c b}\right|$ \\
\hline $\mathrm{S} 1 \quad\left(\xi_{t}^{-1 / 3} \xi_{b}\right)\left(\frac{m_{d}}{m_{s}}\right)^{1 / 3}\left(\frac{m_{s}}{m_{b}}\right)^{2 / 3}-\left(\xi_{t} \xi_{b}^{-1 / 3}\right)\left(\frac{m_{u}}{m_{c}}\right)^{1 / 3}\left(\frac{m_{c}}{m_{t}}\right)^{2 / 3}$ \\
$\mathrm{~S} 2 \quad\left(\xi_{t}^{-1 / 4} \xi_{b}^{5 / 4}\right)\left(\frac{m_{d}}{m_{s}}\right)^{3 / 8}\left(\frac{m_{s}}{m_{b}}\right)^{3 / 4}-\left(\xi_{t}^{5 / 4} \xi_{b}^{-1 / 4}\right)\left(\frac{m_{u}}{m_{c}}\right)^{3 / 8}\left(\frac{m_{c}}{m_{t}}\right)^{3 / 4}$ \\
A1 \\
A2 \\
$\left(\xi_{t}^{-1 / 4} \xi_{b}^{5 / 4}\right)\left(\frac{m_{d}}{m_{s}}\right)^{3 / 8}\left(\frac{m_{s}}{m_{b}}\right)^{3 / 4}$ \\
$\left(\xi_{t}^{-1 / 4} \xi_{b}^{5 / 4}\right)\left(\frac{m_{d}}{m_{s}}\right)^{3 / 8}\left(\frac{m_{s}}{m_{b}}\right)^{3 / 4}$ \\
\hline \hline
\end{tabular}

$\left|V_{c b}\right|_{\exp }=0.039 \pm 0.003$ [31). The similar problem exists for the renormalized $\left|V_{u b} / V_{c b}\right|$ and $\left|V_{t d} / V_{t s}\right|$ in Pattern A2. Hence it is difficult, even impossible, to numerically "justify" the proposed mass matrix patterns through their consequences on $\left|V_{c b}\right|$, before the value of $\tan \beta$ in the MSSM can be reliably fixed somewhere else.

(2) With the inputs taken above and appropriate values of $\tan \beta$, we find that only Pattern $\mathrm{S} 1$ is likely to properly reproduce $\left|V_{c b}\right|$ at the weak scale [10]. Of course the other three patterns could also lead to proper results of $\left|V_{c b}\right|$, if one changes the input values of quark mass ratios and takes their large errors into account. The interesting point is that the value of the renormalized $\left|V_{c b}\right|$ in Pattern S1 is remarkably larger than those in Patterns S2, A1 and A2. Therefore it is in principle possible to distinguish Pattern S1 from the others through the window of $\left|V_{c b}\right|$. Numerically, however, it is extremely difficult to distinguish between Patterns S2 and A1.

(3) Pattern A2 can be ruled out if it fails in reproducing the experimental values of $\left|V_{c b}\right|$ and $\left|V_{u b} / V_{c b}\right|$, simultaneously, with suitable inputs of quark mass ratios. In view of the illustrative Figs. 1 and 2, we find that both $\left|V_{c b}\right|$ and $\left|V_{u b} / V_{c b}\right|$ obtained from Pattern A2 are not favored by current data for all possible values of $\tan \beta$. Indeed this pattern is less attractive than the others due to its complicated analytical consequences on $\left|V_{u b} / V_{c b}\right|$ and $\left|V_{t d} / V_{t s}\right|$, as we have pointed out before. 


\section{Concluding remarks}

We have studied a few typical patterns of quark mass matrices within the framework of orbifold models. Two of the five RRR-type mass matrices have been derived, but it is difficult to obtain the others from $Z_{6}-$ II orbifold models. One of the main difficulties is that one cannot generate several hierarchies in a quark mass matrix by only using selection rules of orbifold models, because the number of twisted sectors is limited. This is a remarkable difference of our approach from that with gauge symmetries, where any charge to generate more hierarchies could be assigned. In addition, we have derived two types of realistic quark mass matrices with four texture zeros and up-down structural parallelism.

For the purpose of illustration, we have confronted the obtained results of quark flavor mixings at the string scale with low-energy data by use of the renormalization-group equations. With the same input values of quark mass ratios, we find that the four mass matrix patterns proposed above cannot all be in good agreement with current experimental data. It is worth

emphasizing that some instructive scale-independent relations like $\left|V_{u b} / V_{c b}\right| \approx \sqrt{m_{u} / m_{c}}$ and $\left|V_{t d} / V_{t s}\right| \approx \sqrt{m_{d} / m_{s}}$, which are favored by current experimental data, might imply a right way in phenomenology towards the correct pattern of quark mass matrices at the superstring scale.

One can extend the analyses made in this work so as to obtain other types of quark mass matrices. Also it should be interesting to apply our approach to the lepton mass matrices.

\section{Acknowledgments}

One of us (ZZX) would like to thank H. Fritzsch for his helpful comments on the stringinspired quark mass Ansätze. He is also grateful to A.I. Sanda for his warm hospitality and to the Japan Society for the Promotion of Science for its financial support. 


\section{References}

[1] C.D. Froggatt and H.B. Nielsen, Nucl. Phys. B 147, 277 (1979); S. Dimopoulos, Phys. Lett. B 129, 417 (1983).

[2] L.E. Ibáñez and G.G. Ross, Phys. Lett. B 332, 100 (1994).

[3] M. Leurer, Y. Nir and N. Seiberg, Nucl. Phys. B 398, 319 (1993); Nucl. Phys. B 420, 468 (1994); Y. Nir and N. Seiberg, Phys. Lett. B 309, 337 (1993); V. Jain and R. Shrock, Phys. Lett. B 352, 83 (1995); P. Binétruy and P. Ramond, Phys. Lett. B 350, 49 (1995); E. Dudas, S. Pokorski and C.A. Savoy, Phys. Lett. B 356, 45 (1995).

[4] M. Drees, Phys. Lett. B 181, 279 (1986); J.S. Hagelin and S. Kelley, Nucl. Phys. B 342, 95 (1990); A.E. Faraggi, J.S. Hagelin, S. Kelley and D.V. Nanopoulos, Phys. Rev. D 45, 3272 (1992); Y. Kawamura, Phys. Rev. D 53, 3779 (1996); Y. Kawamura and T. Kobayashi, Phys. Lett. B 375, 141 (1996); Report No. INS-Rep-1153 hepph/9608233); Y. Kawamura, T. Kobayashi and T. Komatsu, Report No. INS-Rep-1161 (hep-ph/9609462), to be published in Phys. Lett. B.

[5] J. Ellis and D.V. Nanopoulos, Phys. Lett. B 110, 44 (1982); R. Barbieri and R. Gatto, Phys. Lett. B 110, 211 (1982); T. Inami and C.S. Lim, Nucl. Phys. B 207, 533 (1982); J. Hagelin, S. Kelly and T. Tanaka, Nucl. Phys. B 415, 293 (1994).

[6] J.L. Lopez and D.V. Nanopoulos, Nucl. Phys. B 338, 73 (1990); A.E. Faraggi and E. Halyo, Nucl. Phys. B 416, 63 (1994).

[7] N. Haba, C. Hattori, M. Matsuda, and T. Matsuoka, Prog. Theor. Phys. 96, 1249 (1996).

[8] K.S. Babu and R.N. Mohapatra, Phys. Rev. Lett. 74, 2418 (1995).

[9] T. Kobayashi, Phys. Lett. B 358, 253 (1995).

[10] T. Kobayashi and Z.Z. Xing, Mod. Phys. Lett. A 12, 561 (1997).

[11] M. Cvetic, Phys. Rev. Lett. 59, 1795 (1987); A. Font, L.E. Ibáñez, H.P. Nilles and F. Quevedo, Phys. Lett. B 213, 274 (1988).

[12] T. Kobayashi, Phys. Lett. B 354, 264 (1995).

[13] R. Ramond, R.G. Roberts and G.G. Ross, Nucl. Phys. B 406, 19 (1993).

[14] L. Dixon, J. Harvey, C. Vafa and E. Witten, Nucl. Phys. B 261, 678 (1985); Nucl. Phys. B 274, 285 (1986); L.E. Ibáñez, J. Mas, H.P. Nilles and F. Quevedo, Nucl. Phys. B 301, 157 (1988); Y. Katsuki, Y. Kawamura, T. Kobayashi, N. Ohtsubo, Y. Ono and K. Tanioka, Nucl. Phys. B 341, 611 (1990). 
[15] T. Kobayashi and N. Ohtsubo, Phys. Lett. B 245, 441 (1990).

[16] T. Kobayashi and N. Ohtsubo, Int. J. Mod. Phys. A 9, 87 (1994).

[17] D. Friedan, E. Martinec and S. Shenker, Nucl. Phys. B 271, 93 (1986).

[18] S. Hamidi and C. Vafa, Nucl. Phys. B 279, 465 (1987); L. Dixon, D. Friedan, E. Martinec and S. Shenker, Nucl. Phys. B 282, 13 (1987).

[19] Y. Katsuki, Y. Kawamura, T. Kobayashi, N. Ohtsubo, Y. Ono and K. Tanioka, Phys. Lett. B 218, 169 (1989); Y. Kawamura and T. Kobayashi, Nucl. Phys. B 481, 539 (1996).

[20] H. Fritzsch, Phys. Lett. B 73, 317 (1978); Nucl. Phys. B 155, 189 (1979).

[21] J. Erler, D. Jungnickel and J. Lauer, Phys. Rev. D 45, 3651 (1992); D. Jungnickel, J. Lauer, M. Spaliński and S. Stieberger, Mod. Phys. Lett. A 7, 3059 (1992); J. Erler, D. Jungnickel, M. Spaliński and S. Stieberger, Nucl. Phys. B 397, 379 (1993).

[22] C.S. Lim, Phys. Lett. B 256, 233 (1991); M. Dine R.G. Leigh and D.A. MacIntire, Phys. Rev. Lett. 69, 2030 (1992); K. Choi, D.B. Kaplan and A.E. Nelson, Nucl. Phys. B 391, 515 (1993); T. Kobayashi and C.S. Lim, Phys. Lett. B 343, 122 (1995).

[23] See, e.g., Z.Z. Xing, J. Phys. G 23, 717 (1997).

[24] Particle Data Group, R.M. Barnett et al., Phys. Rev. D 54, 1 (1996).

[25] Z.Z. Xing, Nucl. Phys. B (Proc. Suppl.) 50, 24 (1996); Nuovo Cimento A 109, 115 (1996).

[26] K.S. Babu and Q. Shafi, Phys. Rev. D 47, 5004 (1993).

[27] H. Leutwyler, Phys. Lett. B 378, 313 (1996); and private communications.

[28] H. Fritzsch and Z.Z. Xing, Phys. Lett. B 353, 114 (1995); Phys. Lett. B 413, 396 (1997); hep-ph/9707215 and hep-ph/9708366.

[29] See, e.g., L.J. Hall and A. Rasin, Phys. Lett. B 315, 164 (1993); D. Du and Z.Z. Xing, Phys. Rev. D 48, 2349 (1993); S.S. Xue, Phys. Lett. B 398, 177 (1997); K. Harayama, N. Okamura, A.I. Sanda, and Z.Z. Xing, Prog. Theor. Phys. 97, 781 (1997).

[30] J. Gasser and H. Leutwyler, Phys. Rep. C 87, 77 (1982).

[31] A. Ali and D. London, Report No. DESY 96-140 (talk presented at the QCD Euroconference 96, Montpellier, July, 1996).

[32] Z.Z. Xing, J. Phys. G 23, 1563 (1997); hep-ph/9609204. 


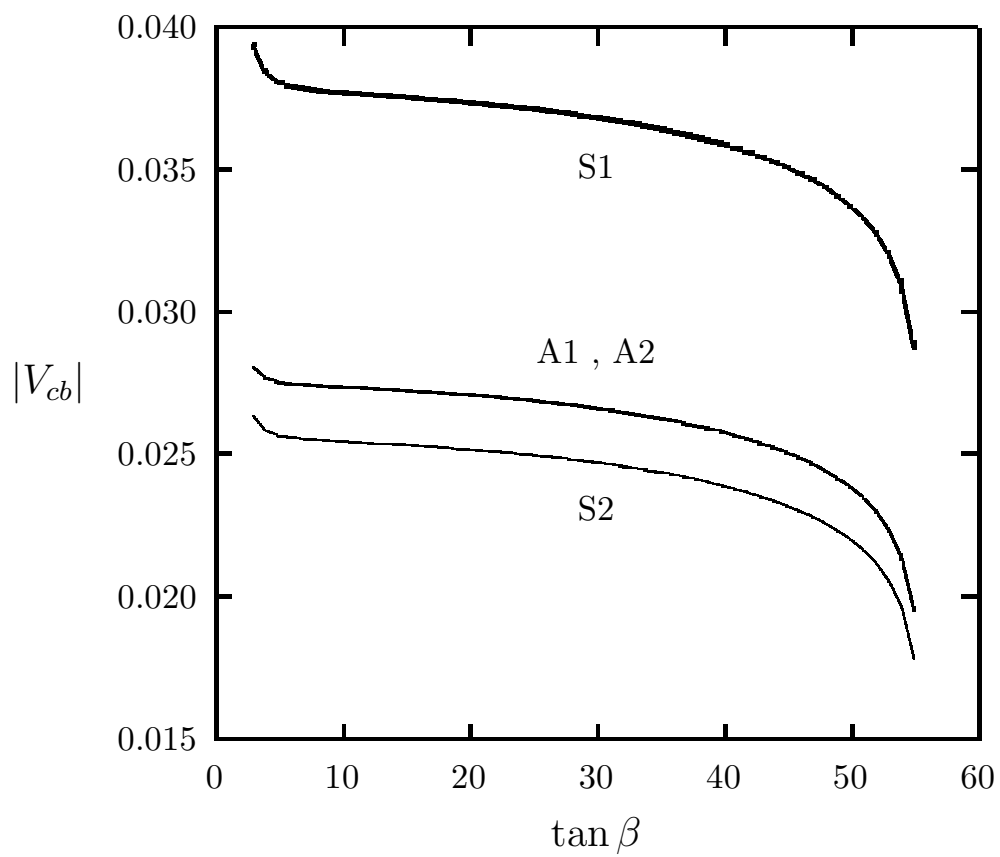

Figure 1: Illustrative plot for $\left|V_{c b}\right|$ changing with $\tan \beta$ at the weak scale $M_{Z}$, obtained from the patterns of quark mass matrices S1, S2, A1 and A2.

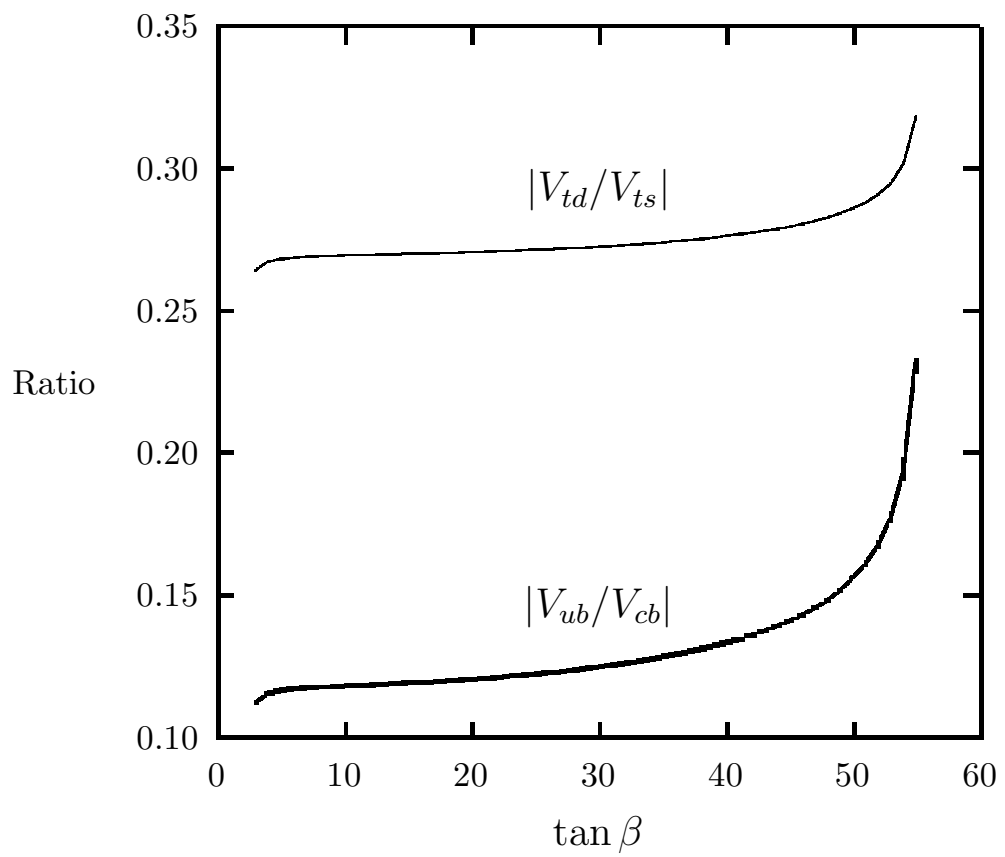

Figure 2: Illustrative plot for the ratios $\left|V_{u b} / V_{c b}\right|$ and $\left|V_{t d} / V_{t s}\right|$ changing with tan $\beta$ at the weak scale $M_{Z}$, obtained from the quark mass matrix pattern A2 (here $\phi=\pi / 2$ has been taken). 